Check for updates

Cite this: RSC Adv., 2017, 7, 28009

Received 30th March 2017

Accepted 23rd May 2017

DOI: $10.1039 / \mathrm{c7ra03588h}$

rsc.li/rsc-advances

\section{Semisynthesis of esters and oxime esters/ sulfonates from furyl-ring-based acetylation derivatives of fraxinellone as insecticidal agents $\uparrow$}

\author{
Yong Guo, iD $t^{a}$ Ruige Yangt $t^{a}$ and Hui Xu iD *ab
}

In continuation of our program to discover new natural-product-based crop protection agents, we prepared a series of esters and oxime esters/sulfonates from furyl-ring-based acetylation derivatives as pesticidal agents by structural modification of fraxinellone, a biorenewable degraded limonoid isolated from Meliaceae and Rutaceae plants. The structural assignment was based on the spectroscopic and Xray analysis data. Their insecticidal activity was evaluated against a crop-threatening agricultural insect pest, Mythimna separata Walker. Among all derivatives, compounds 9a and 10i exhibited the most promising pesticidal activity. Their structure-activity relationships were also discussed.

\section{Introduction}

Oriental armyworm (Mythimna separata Walker) is a terrible lepidopteran insect pest, and its infestations are hard to control. ${ }^{1,2}$ In 2012, approximately 4 million hectares of crops were completely lost in China due to the intermittent outbreaks of $M$. separata. ${ }^{3}$ Obviously, lots of chemical pesticides had to be extensively applied to deal with insect pest outbreaks, however, it ultimately led to insect pests resistance, and human health and environmental problems. ${ }^{4-7}$ Consequently, research and development of new potential alternatives to effectively and selectively control insect pests is extremely desirable.

Fraxinellone (1, Fig. 1), a biorenewable degraded limonoid, is isolated from many Meliaceae and Rutaceae plants, and exhibits a variety of interesting properties, including the antiinflammatory bowel disease, ${ }^{8}$ neuroprotective, ${ }^{9}$ and insecticidal activities..$^{10-13}$ In our previous reports, compound 1 was modified at its C-4/C-10 positions, and some fraxinellone-based oxime esters $^{\mathbf{1 4}}$ (I and II, Fig. 1) and $\operatorname{esters}^{\mathbf{1 5}}$ (III and IV, Fig. 1) displayed higher insecticidal activity than toosendanin against pre-third-instar larvae of Mythimna separata. In addition, to the best of our knowledge, little attention has been paid to the structural modifications on the C-ring (furyl-ring) of compound $\mathbf{1}$ as pesticidal agents. Based upon the above results, and in

\footnotetext{
${ }^{a}$ Research Institute of Pesticidal Design \& Synthesis, College of Chemistry \& Pharmacy, Northwest A\&F University, Yangling 712100, Shaanxi Province, China. E-mail: orgxuhui@nwsuaf.edu.cn; Fax: +86-29-87091952; Tel: +86-29-87091952

${ }^{b}$ Shaanxi Key Laboratory of Natural Products \& Chemical Biology, Northwest A\&F University, Yangling 712100, Shaanxi Province, China

$\dagger$ Electronic supplementary information (ESI) available: Characterization for the new compounds. CCDC 1524889-1524891, 1524894 and 1524893. For ESI and crystallographic data in CIF or other electronic format see DOI: 10.1039/c7ra03588h

\$ These authors contributed equally to this work.
}

continuation of our program to discover biorenewable fraxinellone-based pesticides, herein we wanted to prepare a series of new esters/oxime esters from furyl-ring-based acetylation derivatives of fraxinellone ( $\mathbf{V}$ and VI, Fig. 1) as insecticidal agents against $M$. separata in vivo.

\section{Materials and methods}

\section{General}

All chemical reagents were purchased and utilized without further purification. Melting point (mp) was determined using the XT-4 digital mp apparatus. Optical rotation was measured using an Autopol III automatic polarimeter. Infrared (IR) spectra were measured by a TENSOR 27 spectrometer. Proton nuclear magnetic resonance spectra $\left({ }^{1} \mathrm{H}\right.$ NMR) were measured with the Avance 400 or $500 \mathrm{MHz}$ equipment. 5'-Acetylfraxinellone (2) and $2^{\prime}$-acetylfraxinellone (3) were prepared according to our previous paper. ${ }^{16}$

\section{Synthesis of compound 4}

To a stirred solution of $2(0.5 \mathrm{mmol})$ in methanol at $0-5{ }^{\circ} \mathrm{C}$, $\mathrm{NaBH}_{4}(1 \mathrm{mmol})$ was slowly added. After addition, the reaction mixture was stirred at $0-5{ }^{\circ} \mathrm{C}$ for $1.5 \mathrm{~h}$. Then the solvent was removed, and the residue was dissolved in $\mathrm{CH}_{2} \mathrm{Cl}_{2}(50 \mathrm{~mL})$. The mixture was washed by brine $(20 \mathrm{~mL})$. The organic phase was dried over anhydrous $\mathrm{Na}_{2} \mathrm{SO}_{4}$, filtered, and concentrated under reduced pressure. Finally, the crude product was purified by preparative thin-layer chromatography (PTLC) to give 4 as a white solid in $72 \%$ yield.

Data for 4 (isomer $\alpha: \beta=1: \mathbf{1})$. Mp $96-98{ }^{\circ} \mathrm{C} ;[\alpha]_{\mathrm{D}}^{20}=-27(c$ $3.0 \mathrm{mg} \mathrm{mL}^{-1}$, acetone); IR $\mathrm{cm}^{-1}: 3402,2933,2896,1748,1673$, 1671, 1205, 978; ${ }^{1} \mathrm{H}$ NMR (400 MHz, $\left.\mathrm{CDCl}_{3}\right) \delta: 7.40\left(\mathrm{~s}, 1 \mathrm{H}, \mathrm{H}-2^{\prime}\right)$, $6.20\left(\mathrm{~s}, 1 \mathrm{H}, \mathrm{H}-4^{\prime}\right), 4.86-4.89\left(\mathrm{~m}, 1 \mathrm{H},-\mathrm{OH}(\mathrm{CH}) \mathrm{CH}_{3}\right), 4.83(\mathrm{~s}, 1 \mathrm{H}$, 


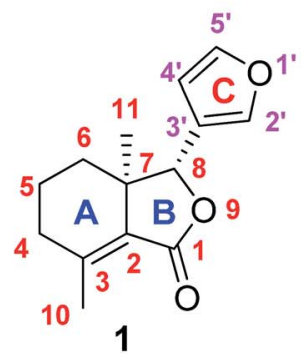<smiles>[R]C(=O)O/N=C1/CC[C@@]2(C)C(=C1C)C(=O)O[C@H]2c1ccoc1</smiles><smiles>[R]C(=O)O/N=C/C1=C2C(=O)O[C@@H](c3ccoc3)[C@@]2(C)CCC1</smiles><smiles>[R]C(=O)O[C@H]1CC[C@]2(C)C(=C1C)C(=O)O[C@H]2c1ccoc1</smiles><smiles>[R]C(=O)OCC1=C2C(=O)O[C@@H](c3ccoc3)[C@@]2(C)CCC1</smiles>

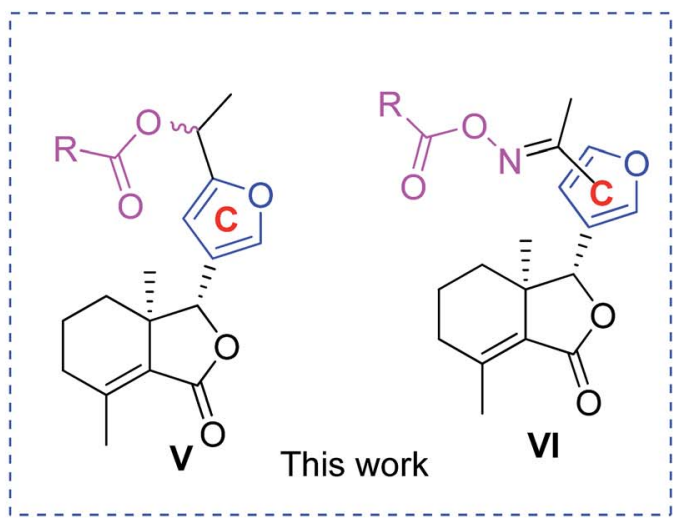

Fig. 1 Chemical structures of fraxinellone (1), and its derivatives (I-VI).

H-8), 2.12-2.31 (m, 2H, H-4), 2.11 (s, 3H, H-10), $2.03(\mathrm{~s}, 1 \mathrm{H}$, $-\mathrm{OH}), 1.71-1.87$ (m, 3H, H-5, 6), $1.55\left(\mathrm{~s}, 1.5 \mathrm{H},-\mathrm{OH}(\mathrm{CH}) \mathrm{CH}_{3}\right)$, $1.53\left(\mathrm{~s}, 1.5 \mathrm{H},-\mathrm{OH}(\mathrm{CH}) \mathrm{CH}_{3}\right), 1.40-1.46(\mathrm{~m}, 1 \mathrm{H}, \mathrm{H}-6), 0.87(\mathrm{~s}, 3 \mathrm{H}$, $\mathrm{H}-11)$. HRMS (ESI): calcd for $\mathrm{C}_{16} \mathrm{H}_{21} \mathrm{O}_{4}\left([\mathrm{M}+\mathrm{H}]^{+}\right), 277.1434$; found, 277.1433 .

\section{General procedure for synthesis of compounds 5 and 6}

A mixture of 2 or $3(0.5 \mathrm{mmol})$, hydroxylamine hydrochloride $(0.75 \mathrm{mmol})$, and pyridine $(2 \mathrm{mmol})$ in absolute ethanol $(10 \mathrm{~mL})$ was refluxed. When the reaction was complete according to TLC analysis, the solvent was removed under reduced pressure. Finally, the crude product was purified by PTLC to give 5 (72\% yield) or 6 ( $82 \%$ yield) as a white solid.

Data for 5. Mp $176-178{ }^{\circ} \mathrm{C}$; $[\alpha]_{\mathrm{D}}^{20}=-25\left(\right.$ c $3.8 \mathrm{mg} \mathrm{mL}{ }^{-1}$, acetone); IR $\mathrm{cm}^{-1}$ : 3265, 2961, 2922, 2855, 1746, 1264, 1049, 901; ${ }^{1} \mathrm{H}$ NMR (400 MHz, $\left.\mathrm{CDCl}_{3}\right) \delta: 7.49\left(\mathrm{~s}, 1 \mathrm{H}, \mathrm{H}-2^{\prime}\right), 6.59(\mathrm{~s}, 1 \mathrm{H}$, $\left.\mathrm{H}-4^{\prime}\right), 4.87$ (s, 1H, H-8), 2.17-2.32 (m, 6H, $-\mathrm{CH}_{3},-\mathrm{OH}$ and $\left.\mathrm{H}-4\right)$, 2.13 (s, 3H, H-10), 1.72-1.84 (m, 3H, H-5, 6), 1.42-1.48 (m, 1H, $\mathrm{H}-6), 0.87$ (s, 3H, H-11). HRMS (ESI): calcd for $\mathrm{C}_{16} \mathrm{H}_{20} \mathrm{O}_{4} \mathrm{~N}([\mathrm{M}+$ $\left.\mathrm{H}]^{+}\right), 290.1387$; found, 290.1387 .

Data for 6. Mp $186-188{ }^{\circ} \mathrm{C} ;[\alpha]_{\mathrm{D}}^{20}=88$ (c $4.1 \mathrm{mg} \mathrm{mL}{ }^{-1}$, acetone); IR $\mathrm{cm}^{-1}$ : 3300, 2957, 2925, 2852, 1727, 1670, 1272, 1218, 984; ${ }^{1} \mathrm{H}$ NMR (500 MHz, $\mathrm{CDCl}_{3}$ ) $\delta: 7.43$ (s, 1H, H-5'), 7.31 (s, 1H, -OH), 6.61 (d, $\left.J=1.5 \mathrm{~Hz}, 1 \mathrm{H}, \mathrm{H}-4^{\prime}\right), 5.59$ (s, 1H, H-8), 2.21-2.26 (m, $4 \mathrm{H},-\mathrm{CH}_{3}$ and $\left.\mathrm{H}-4\right), 2.08-2.16(\mathrm{~m}, 4 \mathrm{H}, \mathrm{H}-4,10)$, 1.75-1.77 (m, 1H, H-5), 1.58-1.68 (m, 3H, H-5, 6), 0.92 (s, 3H, H11). HRMS (ESI): calcd for $\mathrm{C}_{16} \mathrm{H}_{20} \mathrm{O}_{4} \mathrm{~N}\left([\mathrm{M}+\mathrm{H}]^{+}\right), 290.1387$; found, 290.1385 .
General procedure for synthesis of compounds $8 b-j, 9 a-j$ and $10 a-j$

A mixture of the corresponding acid (7, $0.28 \mathrm{mmol}), N, N^{\prime}$-dicyclohexylcarbodiimide (DCC, $0.28 \mathrm{mmol}$ ), 4-dimethylaminopyridine (DMAP, $0.04 \mathrm{mmol})$, and 4,5 or $6(0.2 \mathrm{mmol})$ in dry $\mathrm{CH}_{2} \mathrm{Cl}_{2}$ $(10 \mathrm{~mL})$ was stirred at room temperature. When the reaction was complete according to TLC analysis, the mixture was diluted by $\mathrm{CH}_{2} \mathrm{Cl}_{2}(30 \mathrm{~mL})$, washed by $\mathrm{HCl}\left(0.1 \mathrm{~mol} \mathrm{~L}^{-1}, 15 \mathrm{~mL}\right)$, $5 \% \mathrm{NaHCO}_{3}(15 \mathrm{~mL})$ and brine $(15 \mathrm{~mL})$, dried over anhydrous $\mathrm{Na}_{2} \mathrm{SO}_{4}$, concentrated in vacuo, and purified by PTLC to give products $\mathbf{8 b}-\mathbf{j}, \mathbf{9 a}-\mathbf{j}$ and $\mathbf{1 0 a}-\mathbf{j}$. The example data of $\mathbf{8 b}-\mathbf{e}, \mathbf{9 a}-\mathbf{e}$ and $\mathbf{1 0 a}-\mathbf{e}$ were described as follows, whereas the data of $\mathbf{8 f}-\mathbf{j}$, $\mathbf{9 f}-\mathbf{j}$ and $\mathbf{1 0 f}-\mathbf{j}$ were shown in the ESI. $\dagger$

Data for 8b. Yield: $70 \%$, colorless liquid; $[\alpha]_{\mathrm{D}}^{20}=-17(c$ $3.1 \mathrm{mg} \mathrm{mL}{ }^{-1}$, acetone); IR $\mathrm{cm}^{-1}$ : 2951, 2924, 2853, 1754, 1717, 1451, 1265, 1205, 1048; ${ }^{1} \mathrm{H}$ NMR (400 MHz, $\left.\mathrm{CDCl}_{3}\right) \delta: 8.04$ (d, $J=$ $8.0 \mathrm{~Hz}, 2 \mathrm{H},-\mathrm{Ph}), 7.54(\mathrm{t}, \mathrm{d}, J=7.6 \mathrm{~Hz}, 1 \mathrm{H},-\mathrm{Ph}), 7.41-7.45(\mathrm{~m}$, $\left.3 \mathrm{H},-\mathrm{Ph}, \mathrm{H}-2^{\prime}\right), 6.35\left(\mathrm{~s}, 1 \mathrm{H}, \mathrm{H}-4^{\prime}\right), 6.16-6.21\left(\mathrm{~m}, 1 \mathrm{H},-\mathrm{O}(\mathrm{CH}) \mathrm{CH}_{3}\right)$, 4.84 (s, 1H, H-8), 2.24-2.31 (m, 2H, H-4), 2.12 (s, 3H, H-10), 1.79$1.83(\mathrm{~m}, 3 \mathrm{H}, \mathrm{H}-5,6), 1.71$ (d, $\left.J=2.4 \mathrm{~Hz}, 1.5 \mathrm{H},-\mathrm{OH}(\mathrm{CH}) \mathrm{CH}_{3}\right)$, $1.69\left(\mathrm{~d}, J=2.4 \mathrm{~Hz}, 1.5 \mathrm{H},-\mathrm{OH}(\mathrm{CH}) \mathrm{CH}_{3}\right), 1.40-1.47$ (m, $\left.1 \mathrm{H}, \mathrm{H}-6\right)$, 0.86-0.87 (m, 3H, H-11). HRMS (ESI): calcd for $\mathrm{C}_{23} \mathrm{H}_{28} \mathrm{O}_{5} \mathrm{~N}([\mathrm{M}+$ $\left.\mathrm{NH}_{4}\right]^{+}$), 398.1962; found, 398.1959.

Data for 8c. Yield: 81\%, colorless liquid; $[\alpha]_{\mathrm{D}}^{20}=-18($ c 3.2 $\mathrm{mg}$ $\mathrm{mL}^{-1}$, acetone); IR $\mathrm{cm}^{-1}$ : 2952, 2923, 2853, 1756, 1716, 1267, 1047; ${ }^{1} \mathrm{H}$ NMR (400 MHz, $\left.\mathrm{CDCl}_{3}\right) \delta: 7.92$ (d, $\left.J=8.0 \mathrm{~Hz}, 2 \mathrm{H},-\mathrm{Ph}\right)$, $7.43\left(\mathrm{~s}, 1 \mathrm{H}, \mathrm{H}-2^{\prime}\right), 7.22(\mathrm{~d}, J=8.0 \mathrm{~Hz}, 2 \mathrm{H},-\mathrm{Ph}), 6.33(\mathrm{~d}, J=$ $\left.2.4 \mathrm{~Hz}, 1 \mathrm{H}, \mathrm{H}-4^{\prime}\right), 6.14-6.19\left(\mathrm{~m}, 1 \mathrm{H},-\mathrm{O}(\mathrm{CH}) \mathrm{CH}_{3}\right), 4.84(\mathrm{~s}, 1 \mathrm{H}, \mathrm{H}-$ 8), $2.40\left(\mathrm{~s}, 1 \mathrm{H},-\mathrm{PhCH}_{3}\right), 2.17-2.30(\mathrm{~m}, 2 \mathrm{H}, \mathrm{H}-4), 2.12(\mathrm{~s}, 3 \mathrm{H}, \mathrm{H}-$ 
10), $1.73-1.83(\mathrm{~m}, 3 \mathrm{H}, \mathrm{H}-5,6), 1.70(\mathrm{~d}, J=2.4 \mathrm{~Hz}, 1.5 \mathrm{H},-\mathrm{OH}(\mathrm{CH})$ $\left.\mathrm{CH}_{3}\right), 1.68\left(\mathrm{~d}, J=2.4 \mathrm{~Hz}, 1.5 \mathrm{H},-\mathrm{OH}(\mathrm{CH}) \mathrm{CH}_{3}\right), 1.39-1.47(\mathrm{~m}, 1 \mathrm{H}$, $\mathrm{H}-6), 0.86-0.87$ (m, 3H, H-11). HRMS (ESI): calcd for $\mathrm{C}_{24} \mathrm{H}_{30} \mathrm{O}_{5} \mathrm{~N}$ $\left(\left[\mathrm{M}+\mathrm{NH}_{4}\right]^{+}\right), 412.2118$; found, 412.2115.

Data for 8d. Yield: $98 \%$, colorless liquid; $[\alpha]_{\mathrm{D}}^{20}=-18(c$ $3.2 \mathrm{mg} \mathrm{mL}{ }^{-1}$, acetone); IR $\mathrm{cm}^{-1}$ : 2952, 2923, 2853, 1743, 1713, 1606, 1258, 1165; ${ }^{1} \mathrm{H}$ NMR (400 $\left.\mathrm{MHz} \mathrm{CDCl}_{3}\right) \delta: 7.99(\mathrm{~d}, J=$ $9.2 \mathrm{~Hz}, 2 \mathrm{H},-\mathrm{Ph}), 7.43\left(\mathrm{~s}, 1 \mathrm{H}, \mathrm{H}-2^{\prime}\right), 6.89$ (d, $\left.J=8.8 \mathrm{~Hz}, 2 \mathrm{H},-\mathrm{Ph}\right)$, $6.33\left(\mathrm{~s}, 1 \mathrm{H}, \mathrm{H}-4^{\prime}\right), 6.12-6.17\left(\mathrm{~m}, 1 \mathrm{H},-\mathrm{O}(\mathrm{CH}) \mathrm{CH}_{3}\right), 4.84\left(\mathrm{~s}, 1 \mathrm{H}, \mathrm{H}^{-}\right.$ 8), $3.85\left(\mathrm{~s}, 3 \mathrm{H},-\mathrm{OCH}_{3}\right), 2.15-2.31(\mathrm{~m}, 2 \mathrm{H}, \mathrm{H}-4), 2.12(\mathrm{~s}, 3 \mathrm{H}, \mathrm{H}-$ 10), 1.79-1.83 (m, 3H, H-5, 6), 1.69 (d, $J=2.4 \mathrm{~Hz}, 1.5 \mathrm{H}$, $\left.-\mathrm{OH}(\mathrm{CH}) \mathrm{CH}_{3}\right), 1.67$ (d, $\left.J=2.4 \mathrm{~Hz}, 1.5 \mathrm{H},-\mathrm{OH}(\mathrm{CH}) \mathrm{CH}_{3}\right), 1.39-$ 1.47 (m, 1H, H-6), 0.84-0.86 (m, 3H, H-11). HRMS (ESI): calcd for $\mathrm{C}_{24} \mathrm{H}_{27} \mathrm{O}_{6}\left([\mathrm{M}+\mathrm{H}]^{+}\right), 411.1802$; found, 411.1802 .

Data for 8e. Yield: $73 \%$, white solid, mp $158-160{ }^{\circ} \mathrm{C}$; $[\alpha]_{\mathrm{D}}^{20}=$ -23 (c $4.1 \mathrm{mg} \mathrm{mL}^{-1}$, acetone); IR $\mathrm{cm}^{-1}$ : 2953, 2923, 2852, 1755, 1719, 1266, 1048, 855; ${ }^{1} \mathrm{H}$ NMR (400 MHz, $\mathrm{CDCl}_{3}$ ) $\delta: 8.04-8.08$ (m, 2H, -Ph), $7.44\left(\mathrm{~s}, 1 \mathrm{H}, \mathrm{H}-2^{\prime}\right), 7.08-7.12(\mathrm{~m}, 2 \mathrm{H},-\mathrm{Ph}), 6.35(\mathrm{~s}$, $\left.1 \mathrm{H}, \mathrm{H}-4^{\prime}\right), 7.08-7.12(\mathrm{~m}, 2 \mathrm{H},-\mathrm{Ph}), 6.13-6.18(\mathrm{~m}, 1 \mathrm{H},-\mathrm{O}(\mathrm{CH})$ $\left.\mathrm{CH}_{3}\right), 4.84(\mathrm{~s}, 1 \mathrm{H}, \mathrm{H}-8), 2.16-2.31(\mathrm{~m}, 2 \mathrm{H}, \mathrm{H}-4), 2.13(\mathrm{~s}, 3 \mathrm{H}, \mathrm{H}-$ 10), 1.79-1.83 (m, 3H, H-5, 6), $1.70(\mathrm{~d}, J=2.4 \mathrm{~Hz}, 1.5 \mathrm{H}$, $\left.-\mathrm{OH}(\mathrm{CH}) \mathrm{CH}_{3}\right), 1.69$ (d, $\left.J=2.4 \mathrm{~Hz}, 1.5 \mathrm{H},-\mathrm{OH}(\mathrm{CH}) \mathrm{CH}_{3}\right), 1.40-$ 1.47 (m, 1H, H-6), 0.86-0.87 (m, 3H, H-11). HRMS (ESI): calcd for $\mathrm{C}_{23} \mathrm{H}_{27} \mathrm{O}_{5} \mathrm{NF}\left(\left[\mathrm{M}+\mathrm{NH}_{4}\right]^{+}\right)$, 416.1868; found, 416.1862.

Data for 9a. Yield: $78 \%$, white solid, $\operatorname{mp} 89-90{ }^{\circ} \mathrm{C} ;[\alpha]_{\mathrm{D}}^{20}=-23$ (c $4.0 \mathrm{mg} \mathrm{mL} \mathrm{m}^{-1}$, acetone); IR cm $\mathrm{cm}^{-1}$ : 2940, 2873, 1755, 1675, 1205, 1046, 909; ${ }^{1} \mathrm{H}$ NMR (500 MHz, $\left.\mathrm{CDCl}_{3}\right) \delta: 8.04$ (d, $J=8.5 \mathrm{~Hz}, 1 \mathrm{H}$, $\mathrm{Ar}-\mathrm{H}), 8.86$ (d, $J=7.5 \mathrm{~Hz}, 1 \mathrm{H}, \mathrm{Ar}-\mathrm{H}), 7.80$ (d, $J=8.0 \mathrm{~Hz}, 1 \mathrm{H}, \mathrm{Ar}-$ $\mathrm{H}), 7.43-7.56\left(\mathrm{~m}, 5 \mathrm{H}, \mathrm{Ar}-\mathrm{H}\right.$ and $\left.\mathrm{H}-2^{\prime}\right), 6.81\left(\mathrm{~s}, 1 \mathrm{H}, \mathrm{H}-4^{\prime}\right), 4.82(\mathrm{~s}$, $1 \mathrm{H}, \mathrm{H}-8), 4.27$ (s, 2H, $-\mathrm{CH}_{2} \mathrm{C}_{10} \mathrm{H}_{7}$ ), 2.14-2.29 (m, 2H, H-4), 2.12 (s, $3 \mathrm{H},-\mathrm{CH}_{3}$ ), 2.02 (s, 3H, H-10), 1.77-1.85 (m, 2H, H-5, 6), 1.69-1.73 (m, 1H, H-5), 1.39-1.44 (m, 1H, H-6), 0.80 (s, 3H, H-11). HRMS (ESI): calcd for $\mathrm{C}_{28} \mathrm{H}_{28} \mathrm{O}_{5} \mathrm{~N}\left([\mathrm{M}+\mathrm{H}]^{+}\right)$, 458.1962; found, 458.1962.

Data for 9b. Yield: $72 \%$, white solid, mp 200-202 ${ }^{\circ} \mathrm{C}$; $[\alpha]_{\mathrm{D}}^{20}=$ -10 (c $3.4 \mathrm{mg} \mathrm{mL}^{-1}$, acetone); IR $\mathrm{cm}^{-1}$ : 2926, 2853, 1743, 1676, 1250, 1048, 907; ${ }^{1} \mathrm{H}$ NMR (500 MHz, $\left.\mathrm{CDCl}_{3}\right) \delta: 8.10(\mathrm{dd}, J=8.0$, $1.5 \mathrm{~Hz}, 2 \mathrm{H}, \mathrm{Ar}-\mathrm{H}), 7.60-7.63\left(\mathrm{~s}, 2 \mathrm{H}, \mathrm{Ar}-\mathrm{H}\right.$ and $\left.\mathrm{H}-2^{\prime}\right), 7.48(\mathrm{t}, J=$ $8.0 \mathrm{~Hz}, 2 \mathrm{H}, \mathrm{Ar}-\mathrm{H}), 6.97$ (s, 1H, H-4') 4.89 (s, 1H, H-8), 2.46 (s, $\left.3 \mathrm{H},-\mathrm{CH}_{3}\right), 2.27-2.32(\mathrm{~m}, 1 \mathrm{H}, \mathrm{H}-4), 2.18-2.21$ (m, 1H, H-4), 2.14 (s, 3H, H-10), 1.84-1.87 (m, 2H, H-5, 6), 1.73-1.77 (m, 1H, H-5), 1.44-1.49 (m, 1H, H-6), 0.87 (s, 3H, H-11). HRMS (ESI): calcd for $\mathrm{C}_{23} \mathrm{H}_{24} \mathrm{O}_{5} \mathrm{~N}\left([\mathrm{M}+\mathrm{H}]^{+}\right)$, 394.1649; found, 394.1648.

Data for 9c. Yield: $64 \%$, white solid, mp $160-162{ }^{\circ} \mathrm{C}$; $[\alpha]_{\mathrm{D}}^{20}=$ -28 (c $3.4 \mathrm{mg} \mathrm{mL}^{-1}$, acetone); IR $\mathrm{cm}^{-1}: 2940,2872,1745,1675$, 1260, 1049, 911; ${ }^{1} \mathrm{H}$ NMR (500 MHz, $\left.\mathrm{CDCl}_{3}\right) \delta: 7.99(\mathrm{~d}, J=8.5 \mathrm{~Hz}$, $2 \mathrm{H}, \mathrm{Ar}-\mathrm{H}), 7.61$ (s, $\left.1 \mathrm{H}, \mathrm{H}-2^{\prime}\right), 7.28$ (d, $\left.J=8.5 \mathrm{~Hz}, 2 \mathrm{H}, \mathrm{Ar}-\mathrm{H}\right), 6.96$ (s, 1H, H-4'), $4.88(\mathrm{~s}, 1 \mathrm{H}, \mathrm{H}-8), 2.45\left(\mathrm{~s}, 3 \mathrm{H},-\mathrm{PhCH}_{3}\right), 2.44(\mathrm{~s}, 3 \mathrm{H}$, $-\mathrm{CH}_{3}$ ), 2.26-2.32 (m, 1H, H-4), 2.18-2.21 (m, 1H, H-4), 2.14 (s, $3 \mathrm{H}, \mathrm{H}-10), 1.85-1.87$ (m, 2H, H-5, 6), 1.73-1.79 (m, 1H, H-5), 1.45-1.49 (m, 1H, H-6), 0.87 (s, 3H, H-11). HRMS (ESI): calcd for $\mathrm{C}_{24} \mathrm{H}_{26} \mathrm{O}_{5} \mathrm{~N}\left([\mathrm{M}+\mathrm{H}]^{+}\right)$, 408.1805; found, 408.1804.

Data for 9d. Yield: $73 \%$, white solid, mp 150-152 ${ }^{\circ} \mathrm{C}$; $[\alpha]_{\mathrm{D}}^{20}=$ -21 (c $3.2 \mathrm{mg} \mathrm{mL}^{-1}$, acetone); IR $\mathrm{cm}^{-1}$ : 2954, 2923, 2853, 1739, 1607, 1265, 1047, 957; ${ }^{1} \mathrm{H}$ NMR $\left(500 \mathrm{MHz}, \mathrm{CDCl}_{3}\right) \delta: 8.06(\mathrm{~d}, J=$ $9.0 \mathrm{~Hz}, 2 \mathrm{H}, \mathrm{Ar}-\mathrm{H}), 7.62\left(\mathrm{~s}, 1 \mathrm{H}, \mathrm{H}-2^{\prime}\right), 6.96(\mathrm{~d}, J=9.0 \mathrm{~Hz}, 2 \mathrm{H}, \mathrm{Ar}-$ $\mathrm{H}), 6.95\left(\mathrm{~s}, 1 \mathrm{H}, \mathrm{H}-4^{\prime}\right), 4.88$ (s, 1H, H-8), 3.88 (s, 3H, $\left.-\mathrm{OCH}_{3}\right), 2.44$ (s, 3H, - $\left.\mathrm{CH}_{3}\right), 2.17-2.32$ (m, 2H, H-4), 2.14 (s, 3H, H-10), 1.84-
1.88 (m, 2H, H-5, 6), 1.74-1.77 (m, 1H, H-5), 1.44-1.49 (m, 1H, $\mathrm{H}-6), 0.87$ (s, 3H, H-11). HRMS (ESI): calcd for $\mathrm{C}_{24} \mathrm{H}_{26} \mathrm{O}_{6} \mathrm{~N}([\mathrm{M}+$ $\mathrm{H}]^{+}$), 424.1755; found, 424.1754 .

Data for 9e. Yield: $61 \%$, white solid, mp $184-186{ }^{\circ} \mathrm{C}$; $[\alpha]_{\mathrm{D}}^{20}=$ -28 (c $3.3 \mathrm{mg} \mathrm{mL}^{-1}$, acetone); IR $\mathrm{cm}^{-1}: 2934,2871,1748,1673$, 1601, 1257, 1047, 910; ${ }^{1} \mathrm{H}$ NMR (500 MHz, $\left.\mathrm{CDCl}_{3}\right) \delta: 8.12-8.15(\mathrm{~m}$, $2 \mathrm{H}, \mathrm{Ar}-\mathrm{H}), 7.61\left(\mathrm{~s}, 1 \mathrm{H}, \mathrm{H}-2^{\prime}\right), 7.15(\mathrm{t}, J=8.5 \mathrm{~Hz}, 2 \mathrm{H}, \mathrm{Ar}-\mathrm{H}), 6.97(\mathrm{~s}$, $\left.1 \mathrm{H}, \mathrm{H}-4^{\prime}\right), 4.89$ (s, $\left.1 \mathrm{H}, \mathrm{H}-8\right), 2.45\left(\mathrm{~s}, 3 \mathrm{H},-\mathrm{CH}_{3}\right), 2.19-2.32(\mathrm{~m}, 2 \mathrm{H}$, $\mathrm{H}-4), 2.14$ (s, 3H, H-10), 1.85-1.87 (m, 2H, H-5, 6), 1.73-1.77 (m, 1H, H-5), 1.45-1.49 (m, 1H, H-6), 0.87 (s, 3H, H-11). HRMS (ESI): calcd for $\mathrm{C}_{23} \mathrm{H}_{23} \mathrm{O}_{5} \mathrm{NF}\left([\mathrm{M}+\mathrm{H}]^{+}\right), 412.1555$; found, 412.1556 .

Data for 10a. Yield: $75 \%$, white solid, $\mathrm{mp} 70-71^{\circ} \mathrm{C} ;[\alpha]_{\mathrm{D}}^{20}=82$ (c $3.3 \mathrm{mg} \mathrm{mL}^{-1}$, acetone); IR cm ${ }^{-1}: 2952,2924,2853,1752,1207$, 908; ${ }^{1} \mathrm{H}$ NMR $\left(500 \mathrm{MHz}, \mathrm{CDCl}_{3}\right) \delta: 8.01(\mathrm{~d}, J=8.5 \mathrm{~Hz}, 1 \mathrm{H}, \mathrm{Ar}-\mathrm{H})$, 7.87 (d, $J=8.0 \mathrm{~Hz}, 1 \mathrm{H}, \mathrm{Ar}-\mathrm{H}), 7.81-7.82$ (m, 1H, Ar-H), 7.43$7.56\left(\mathrm{~m}, 5 \mathrm{H}, \mathrm{Ar}-\mathrm{H}\right.$ and $\left.\mathrm{H}-5^{\prime}\right), 6.66\left(\mathrm{~d}, J=1.5 \mathrm{~Hz}, 1 \mathrm{H}, \mathrm{H}-4^{\prime}\right), 5.69$ (s, $1 \mathrm{H}, \mathrm{H}-8), 4.22$ (s, 2H, $\left.-\mathrm{CH}_{2} \mathrm{C}_{10} \mathrm{H}_{7}\right), 2.14-2.19$ (m, 2H, H-4), $2.11\left(\mathrm{~s}, 3 \mathrm{H},-\mathrm{CH}_{3}\right), 2.09$ (s, 3H, H-10), 1.87-1.93 (m, 1H, H-5), 1.68-1.72 (m, 1H, H-6), 1.50-1.60 (m, 2H, H-5, 6), 0.88 (s, 3H, $\mathrm{H}-11)$. HRMS (ESI): calcd for $\mathrm{C}_{28} \mathrm{H}_{28} \mathrm{O}_{5} \mathrm{~N}\left([\mathrm{M}+\mathrm{H}]^{+}\right), 458.1962$; found, 458.1956.

Data for 10b. Yield: $80 \%$, white solid, mp $146-148{ }^{\circ} \mathrm{C} ;[\alpha]_{\mathrm{D}}^{20}=$ 110 (c $3.6 \mathrm{mg} \mathrm{mL}^{-1}$, acetone); IR $\mathrm{cm}^{-1}: 2961,2922,2910,1754$, 1449, 1247, 978; ${ }^{1} \mathrm{H}$ NMR (500 MHz, $\left.\mathrm{CDCl}_{3}\right) \delta: 8.08$ (d, $J=7.0 \mathrm{~Hz}$, $2 \mathrm{H}, \mathrm{Ar}-\mathrm{H}), 7.60$ (t, $J=7.5 \mathrm{~Hz}, 1 \mathrm{H}, \mathrm{Ar}-\mathrm{H}), 7.55$ (d, $J=1.5 \mathrm{~Hz}, 1 \mathrm{H}$, $\left.\mathrm{H}-5^{\prime}\right), 7.48$ (t, $\left.J=8.0 \mathrm{~Hz}, 2 \mathrm{H}, \mathrm{Ar}-\mathrm{H}\right), 6.73\left(\mathrm{~d}, J=2.0 \mathrm{~Hz}, 1 \mathrm{H}, \mathrm{H}-4^{\prime}\right)$, $5.85(\mathrm{~s}, 1 \mathrm{H}, \mathrm{H}-8), 2.49$ (s, 3H, - $\left.\mathrm{CH}_{3}\right), 2.21-2.23$ (m, 2H, H-4), 2.14 (s, 3H, H-10), 2.05-2.12 (m, 1H, H-5), 1.80-1.84 (m, 1H, H-6), 1.63-1.66 (m, 2H, H-5, 6), 0.96 (s, 3H, H-11). HRMS (ESI): calcd for $\mathrm{C}_{23} \mathrm{H}_{24} \mathrm{O}_{5} \mathrm{~N}\left([\mathrm{M}+\mathrm{H}]^{+}\right)$, 394.1649; found, 394.1645 .

Data for 10c. Yield: $75 \%$, white solid, $\operatorname{mp~} 174-176{ }^{\circ} \mathrm{C} ;[\alpha]_{\mathrm{D}}^{20}=$ 111 (c $4.3 \mathrm{mg} \mathrm{mL}^{-1}$, acetone); IR $\mathrm{cm}^{-1}: 2954,2923,2851,1745$, 1459, 1376, 1049, 980; ${ }^{1} \mathrm{H}$ NMR (500 MHz, $\left.\mathrm{CDCl}_{3}\right) \delta: 7.96(\mathrm{~d}, J=$ $8.0 \mathrm{~Hz}, 2 \mathrm{H}, \mathrm{Ar}-\mathrm{H}), 7.54$ (d, $\left.J=1.5 \mathrm{~Hz}, 1 \mathrm{H}, \mathrm{H}-5^{\prime}\right), 7.28$ (d, $J=$ $8.0 \mathrm{~Hz}, 2 \mathrm{H}, \mathrm{Ar}-\mathrm{H}), 6.73$ (d, J=1.5 Hz, 1H, H-4'), 5.84 (s, 1H, H-8), $2.48\left(\mathrm{~s}, 3 \mathrm{H},-\mathrm{CH}_{3}\right), 2.44\left(\mathrm{~s}, 3 \mathrm{H},-\mathrm{PhCH}_{3}\right), 2.21-2.24(\mathrm{~m}, 2 \mathrm{H}, \mathrm{H}-4)$, 2.14 (s, 3H, H-10), 2.05-2.09 (m, 1H, H-5), 1.80-1.83 (m, 1H, H6), 1.62-1.66 (m, 2H, H-5, 6), 0.95 (s, 3H, H-11). HRMS (ESI): calcd for $\mathrm{C}_{24} \mathrm{H}_{26} \mathrm{O}_{5} \mathrm{~N}\left([\mathrm{M}+\mathrm{H}]^{+}\right), 408.1805$; found, 408.1802 .

Data for 10d. Yield: $70 \%$, white solid, mp $120-122{ }^{\circ} \mathrm{C}$; $[\alpha]_{\mathrm{D}}^{20}=$ 110 (c $3.4 \mathrm{mg} \mathrm{mL}^{-1}$, acetone); IR $\mathrm{cm}^{-1}: 2954,2924,2853,1747$, 1252, 1049; ${ }^{1} \mathrm{H}$ NMR (500 MHz, $\mathrm{CDCl}_{3}$ ) $\delta: 8.03(\mathrm{~d}, J=9.0 \mathrm{~Hz}, 2 \mathrm{H}$, $\mathrm{Ar}-\mathrm{H}$ ), 7.53 (d, $\left.J=2.0 \mathrm{~Hz}, 1 \mathrm{H}, \mathrm{H}-5^{\prime}\right), 6.96$ (d, $J=8.5 \mathrm{~Hz}, 2 \mathrm{H}, \mathrm{Ar}-$ $\mathrm{H}), 6.72$ (d, $\left.J=1.5 \mathrm{~Hz}, 1 \mathrm{H}, \mathrm{H}-4^{\prime}\right), 5.84(\mathrm{~s}, 1 \mathrm{H}, \mathrm{H}-8), 3.88(\mathrm{~s}, 3 \mathrm{H}$, $\left.-\mathrm{OCH}_{3}\right), 2.48\left(\mathrm{~s}, 3 \mathrm{H},-\mathrm{CH}_{3}\right), 2.21-2.24(\mathrm{~m}, 2 \mathrm{H}, \mathrm{H}-4), 2.14(\mathrm{~s}, 3 \mathrm{H}$, $\mathrm{H}-10), 2.05-2.12$ (m, 1H, H-5), 1.81-1.84 (m, 1H, H-6), 1.62-1.66 (m, 2H, H-5, 6), 0.95 (s, 3H, H-11). HRMS (ESI): calcd for $\mathrm{C}_{24} \mathrm{H}_{26} \mathrm{O}_{6} \mathrm{~N}\left([\mathrm{M}+\mathrm{H}]^{+}\right), 424.1755$; found, 424.1750 .

Data for 10e. Yield: $74 \%$, white solid, mp $142-144{ }^{\circ} \mathrm{C} ;[\alpha]_{\mathrm{D}}^{20}=$ 126 (c $3.0 \mathrm{mg} \mathrm{mL}{ }^{-1}$, acetone); IR $\mathrm{cm}^{-1}: 2947,2929,2871,1747$, 1602, 1250, 1051, 913; ${ }^{1} \mathrm{H}$ NMR (500 MHz, $\left.\mathrm{CDCl}_{3}\right) \delta: 8.09-8.11$ $(\mathrm{m}, 2 \mathrm{H}, \mathrm{Ar}-\mathrm{H}), 7.55$ (d, $\left.J=2.0 \mathrm{~Hz}, 1 \mathrm{H}, \mathrm{H}-5^{\prime}\right), 7.15-7.19(\mathrm{~m}, 2 \mathrm{H}$, $\mathrm{Ar}-\mathrm{H}), 6.73$ (d, $\left.J=1.5 \mathrm{~Hz}, 1 \mathrm{H}, \mathrm{H}-4^{\prime}\right), 5.83$ (s, 1H, H-8), 2.49 (s, 3H, $-\mathrm{CH}_{3}$ ), 2.21-2.24 (m, 2H, H-4), 2.14 (s, 3H, H-10), 2.04-2.10 (m, 1H, H-5), 1.80-1.83 (m, 1H, H-6), 1.62-1.68 (m, 2H, H-5, 6), 0.96 (s, 3H, H-11). HRMS (ESI): calcd for $\mathrm{C}_{23} \mathrm{H}_{23} \mathrm{O}_{5} \mathrm{NF}\left([\mathrm{M}+\mathrm{H}]^{+}\right)$, 412.1555; found, 412.1554. 


\section{General procedure for synthesis of compounds 11 and 12}

To a stirred solution of $\mathrm{NaH}(1.26 \mathrm{mmol})$ in dry tetrahydrofuran (THF, $10 \mathrm{~mL}$ ) at $-15{ }^{\circ} \mathrm{C}$ was slowly added compound 5 or 6 (0.18 $\mathrm{mmol})$. After addition, the reaction mixture was stirred at $-15{ }^{\circ} \mathrm{C}$ for $10 \mathrm{~min}$. Then, benezenesulfochloride $(0.72 \mathrm{mmol})$ were added. When the reaction was complete according to TLC analysis, saturated aqueous $\mathrm{NaHCO}_{3}(15 \mathrm{~mL})$ was added to the mixture, which was extracted with $\mathrm{CH}_{2} \mathrm{Cl}_{2}(3 \times 40 \mathrm{~mL})$. Finally, the combined organic phase was dried over anhydrous $\mathrm{Na}_{2} \mathrm{SO}_{4}$, filtered, concentrated under reduced pressure, and purified by PTLC to give 11 (78\% yield) or $\mathbf{1 2}$ (48\% yield) as a white solid.

Data for 11. Mp $144-146{ }^{\circ} \mathrm{C}$; $[\alpha]_{\mathrm{D}}^{20}=-14\left(\right.$ c $3.3 \mathrm{mg} \mathrm{mL}{ }^{-1}$, acetone); IR $\mathrm{cm}^{-1}$ : 2952, 2924, 2851, 1752, 1450, 1185, 986; ${ }^{1} \mathrm{H}$ NMR (400 MHz, $\mathrm{CDCl}_{3}$ ) $\delta: 8.01$ (d, $\left.J=8.8 \mathrm{~Hz}, 2 \mathrm{H}, \mathrm{Ar}-\mathrm{H}\right), 7.65$ (d, $J=7.6, \mathrm{~Hz}, 1 \mathrm{H}, \mathrm{Ar}-\mathrm{H}), 7.54-7.60\left(\mathrm{~m}, 3 \mathrm{H}, \mathrm{Ar}-\mathrm{H}\right.$ and $\left.\mathrm{H}-2^{\prime}\right), 7.34(\mathrm{~s}$, $\left.1 \mathrm{H}, \mathrm{H}-4^{\prime}\right), 4.89$ (s, $\left.1 \mathrm{H}, \mathrm{H}-8\right), 2.19-2.34\left(\mathrm{~m}, 5 \mathrm{H},-\mathrm{CH}_{3}\right.$ and $\left.\mathrm{H}-4\right)$, 2.15 (s, 3H, H-10), 1.73-1.88 (m, 3H, H-5, 6), 1.49-1.56 (m, 1H, $\mathrm{H}-6), 0.84$ (s, 3H, H-11). HRMS (ESI): calcd for $\mathrm{C}_{22} \mathrm{H}_{24} \mathrm{O}_{6} \mathrm{NS}([\mathrm{M}+$ $\left.\mathrm{H}^{+}\right)$, 430.1319; found, 430.1316 .
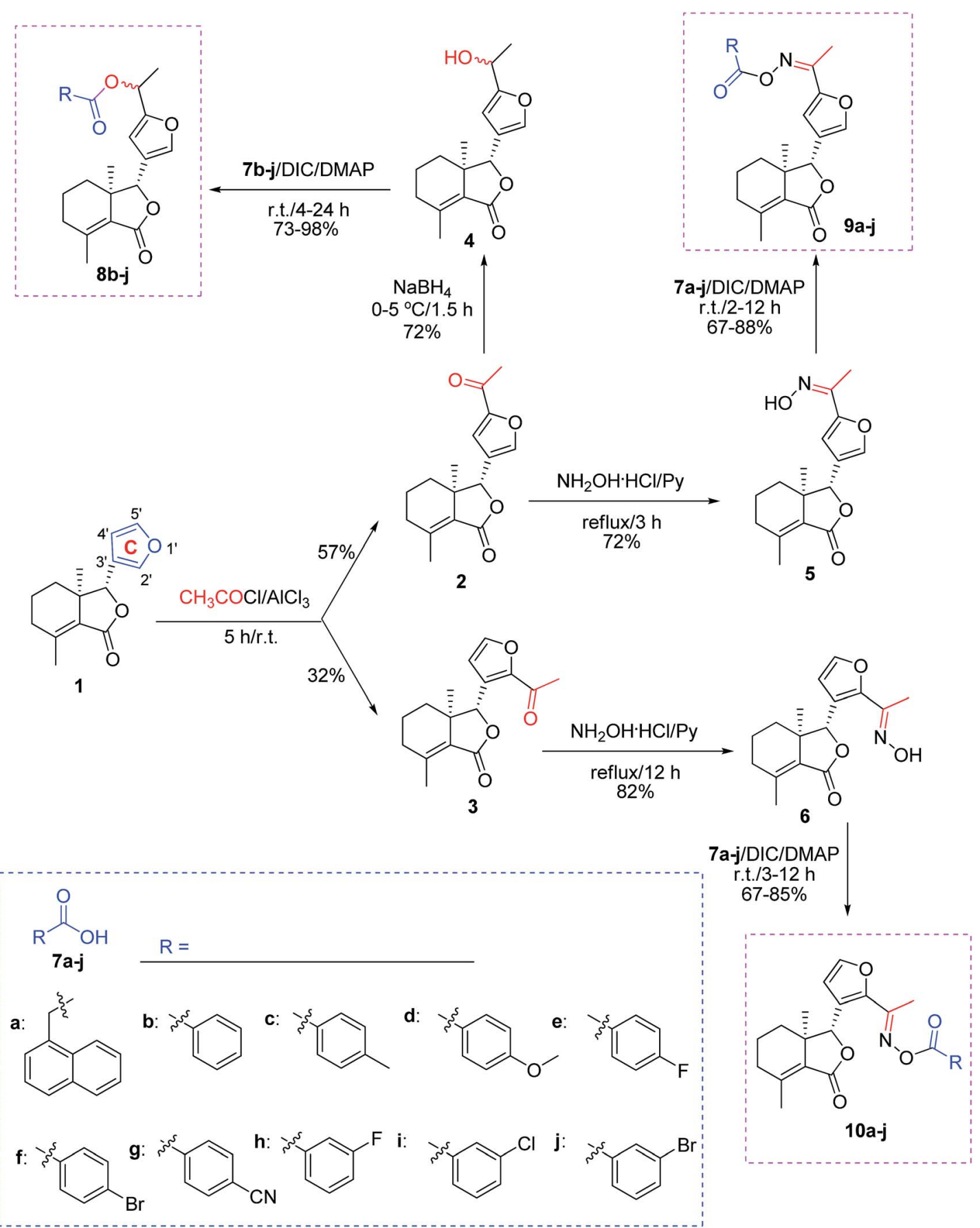

Scheme 1 Synthesis of esters $(8 \mathrm{~b}-\mathrm{j})$ and oxime esters $(9 \mathrm{a}-\mathrm{j}$ and $10 \mathrm{a}-\mathrm{j})$ from furyl-ring-based acetylation derivatives of fraxinellone. 
Data for 12. Mp $100-101{ }^{\circ} \mathrm{C} ;[\alpha]_{\mathrm{D}}^{20}=-5\left(c 4.2 \mathrm{mg} \mathrm{mL}{ }^{-1}\right.$, acetone); IR cm ${ }^{-1}$ : 2955, 2921, 2869, 1750, 1378, 1191, 846; ${ }^{1} \mathrm{H}$ $\operatorname{NMR}\left(500 \mathrm{MHz}, \mathrm{CDCl}_{3}\right) \delta: 8.01(\mathrm{~d}, J=7.5 \mathrm{~Hz}, 2 \mathrm{H}, \mathrm{Ar}-\mathrm{H}), 7.90(\mathrm{~d}$, $J=7.5 \mathrm{~Hz}, 2 \mathrm{H}, \mathrm{Ar}-\mathrm{H}), 7.74(\mathrm{t}, J=7.5 \mathrm{~Hz}, 1 \mathrm{H}, \mathrm{Ar}-\mathrm{H}), 7.66(\mathrm{t}, J=$ $7.5 \mathrm{~Hz}, 1 \mathrm{H}, \mathrm{Ar}-\mathrm{H}), 7.59$ (t, $J=8.0 \mathrm{~Hz}, 2 \mathrm{H}, \mathrm{Ar}-\mathrm{H}), 7.54(\mathrm{t}, J=$ $8.0 \mathrm{~Hz}, 2 \mathrm{H}, \mathrm{Ar}-\mathrm{H}$ ), 7.12 (s, 1H, H-4'), 5.27 (s, 1H, H-8), 2.21-2.26 (m, 2H, H-4), $2.12\left(\mathrm{~s}, 3 \mathrm{H},-\mathrm{CH}_{3}\right), 2.07$ (s, 3H, H-10), 1.78-1.80 (m, 2H, H-5, 6), 1.58-1.61 (m, 1H, H-5), 1.47-1.52 (m, 1H, H-6), 0.57 (s, 3H, H-11). HRMS (ESI): calcd for $\mathrm{C}_{28} \mathrm{H}_{28} \mathrm{O}_{8} \mathrm{NS}_{2}\left([\mathrm{M}+\mathrm{H}]^{+}\right)$, 570.1251; found, 570.1240 .

\section{X-ray crystallography}

Five three-dimensional structures of compounds 4 ( $\alpha-\mathrm{OH}$ isomer), $5,6,9 \mathrm{c}$ and 10e were confirmed by X-ray crystallography. Crystallographic data (excluding structure factors) of 4 ( $\alpha-\mathrm{OH}$ isomer), $5,6,9 \mathrm{c}$ and 10e were deposited at the Cambridge Crystallographic Data Centre (CCDC $\dagger$ ) with deposition numbers of 1524889, 1524890, 1524891, 1524894, and 1524893, respectively.

\section{Biological assay}

Growth inhibitory activity of $1,4-6,8 b-j, 9 a-j, 10 a-j, 11$ and 12 against Mythimna separata. Thirty early 3rd-instar larvae of $M$. separata were chosen as the tested insects for each compound. Solutions of 1, 4-6, 8b-j, 9a-j, 10a-j, 11, 12 and toosendanin (a positive control) were prepared in acetone at $1 \mathrm{mg} \mathrm{mL}^{-1}$. After dipped into the corresponding solution for $3 \mathrm{~s}$, wheat leaf discs (1 $\times 1 \mathrm{~cm}$ ) were taken out and dried. Wheat leaf discs were treated by acetone alone as the blank control group (CK). Several above discs were added to each culture dish (ten insects per dish). Once the discs were consumed, additional ones were added. After $48 \mathrm{~h}$, the rest of compound-soaked discs was cleaned out, and the untreated ones were added till the end of pupae (temperature: 25 $\pm 2{ }^{\circ} \mathrm{C}$; RH: $65-80 \%$; photoperiod: $L / D=12 / 12 \mathrm{~h}$ ). Their corrected mortality rate values were calculated as follows: corrected mortality rate $(\%)=(T-C) \times 100 /(100 \%-C) ; C$ is the mortality rate of CK, and $T$ is the mortality rate of the treated M. separata. ${ }^{15,17}$

\section{Results and discussion}

\section{Synthesis}

As shown in Scheme 1, first, $5^{\prime}$-acetylfraxinellone (2) and $2^{\prime}$-acetylfraxinellone (3) were prepared by reaction of fraxinellone (1) with acetyl chloride in the presence of $\mathrm{AlCl}_{3}{ }^{16}$ Then, reduction of 5 -acetylfraxinellone (2) with $\mathrm{NaBH}_{4}$ gave compound $4(\alpha / \beta=1 / 1$, which was determined by ${ }^{1} \mathrm{H}$ NMR). Hydroxylamine hydrochloride reacted with $\mathbf{2}$ or $\mathbf{3}$ to easily afford the corresponding oximes $\mathbf{5}$ and 6, respectively. Finally, in the presence of $N, N^{\prime}$-dicyclohexylcarbodiimide (DCC) and 4-dimethylaminopyridine (DMAP), the corresponding acid 7 reacted with $\mathbf{4 , 5}$ or $\mathbf{6}$ to produce target products $\mathbf{8 b}-\mathbf{j}, \mathbf{9 a}-\mathbf{j}$ and $\mathbf{1 0 a}-\mathbf{j}$, respectively. On the other hand, as described in Scheme 2, compounds 5 and $\mathbf{6}$ reacting with benezenesulfochloride was also investigated. When $\mathbf{5}$ reacted with benezenesulfochloride ( 4 equiv.), oxime sulfonate 11 was afforded; when 6 reacted with benezenesulfochloride (4 equiv.), interestingly, oxime sulfonate 12 containing $5^{\prime}$-phenylsulfonyl was produced. Their structures were well determined by melting

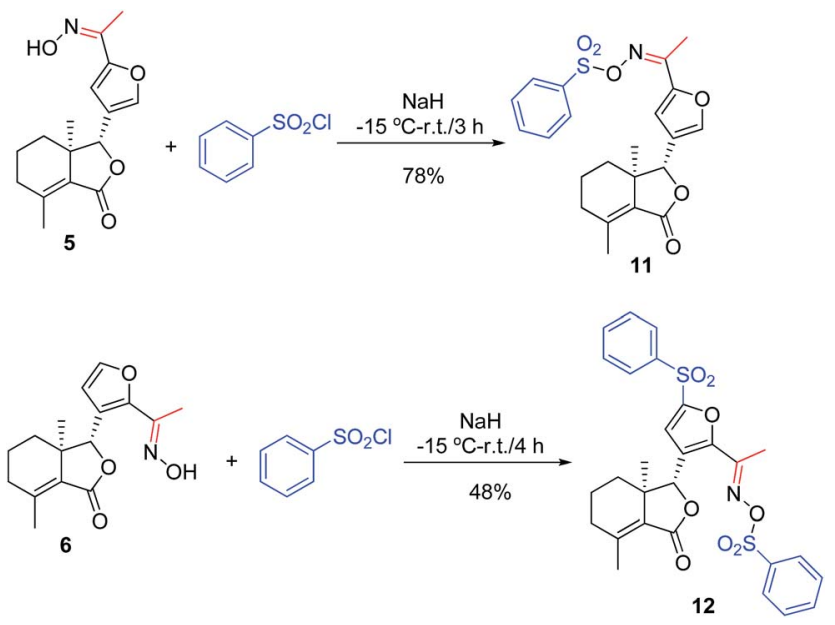

Scheme 2 Synthesis of oxime sulfonates (11 and 12).

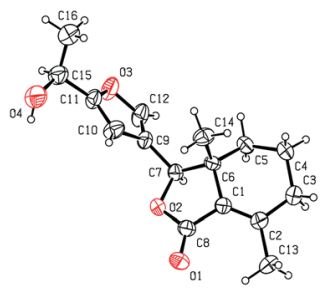

Fig. 2 X-ray crystal structure of compound 4 ( $\alpha$-OH isomer).

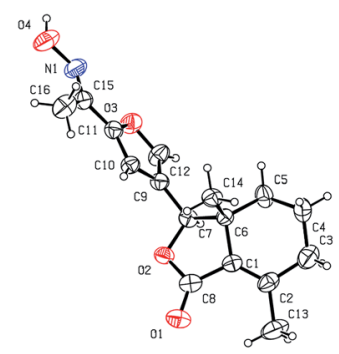

Fig. 3 X-ray crystal structure of compound 5.

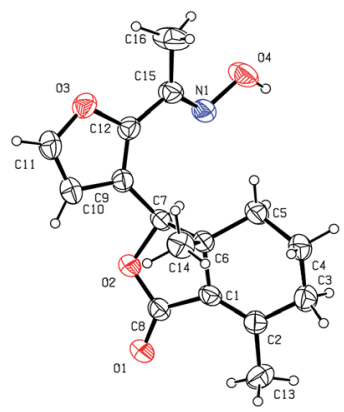

Fig. 4 X-ray crystal structure of compound 6 .

points, optical rotation, IR, and ${ }^{1} \mathrm{H}$ NMR. More importantly, five compounds 4 ( $\alpha-\mathrm{OH}$ isomer), $5,6,9 \mathrm{c}$ and $10 \mathrm{e}$ were further testified by X-ray crystallography (Fig. 2-6). As shown in Fig. 2, the hydroxyethyl was at the $5^{\prime}$-position on the furyl ring, and the $\mathrm{OH}$ 
group was in $\alpha$ position; as shown in Fig. 3 and 4 , the corresponding oxime groups of 5 and $\mathbf{6}$ were at the $5^{\prime}$ and $2^{\prime}$-position on the furyl ring, respectively; as described in Fig. 5 and 6, the 4methylphenylcarbonyloxy of 9c and the 4-fluorophenylcarbonyloxy of 10e were at the $5^{\prime}$ and $2^{\prime}$-position on the furyl ring, respectively.

Comparison of partial ${ }^{1} \mathrm{H}$ NMR spectra of compounds $\mathbf{9 b}$, 10b, 11, and 12 was described in Fig. 7. Assignment of the position of a phenylsulfonyl group on the furyl ring of $\mathbf{1 2}$ was

.

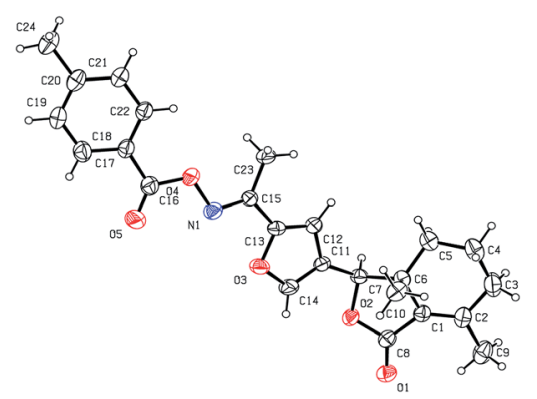

Fig. 5 X-ray crystal structure of compound 9c.

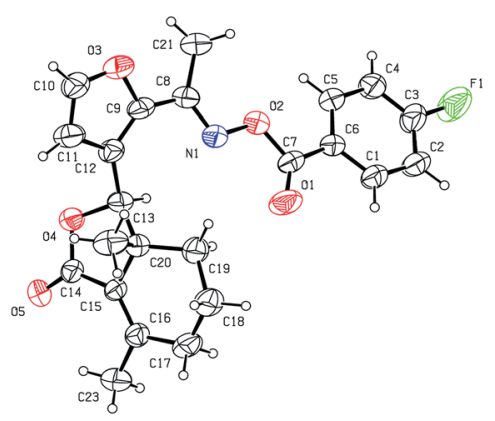

Fig. 6 X-ray crystal structure of compound 10e.

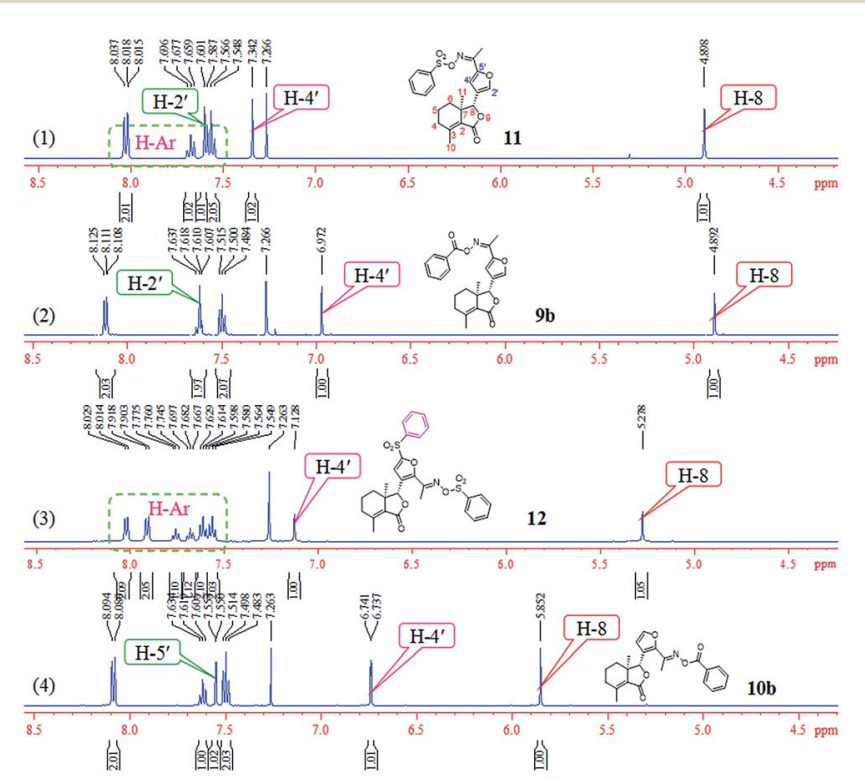

Fig. 7 Comparison of partial ${ }^{1} \mathrm{H}$ NMR spectra of compounds $9 \mathrm{~b}, 10 \mathrm{~b}$, 11 , and 12 . based on the chemical shift. If the phenylsulfonyl group was at the $4^{\prime}$-position on the furyl ring, the corresponding chemical shift of $\mathrm{H}-5^{\prime}$ will greater than $7.342 \mathrm{ppm}$; here the chemical shift was $7.128 \mathrm{ppm}$, which belonged to $\mathrm{H}-4^{\prime}$, so the phenylsulfonyl group was at the $5^{\prime}$-position on the furyl ring of $\mathbf{1 2}$.

\section{Insecticidal activity}

As described in Table 1, the growth inhibitory activity of compounds 1, 4-6, 8b-j, 9a-j, 10a-j, 11 and 12 against $M$. separata was tested at $1 \mathrm{mg} \mathrm{mL}^{-1}$. Toosendanin, a commercial insecticide derived from Melia azedarach, was used as the positive control. Leaves treated with acetone alone were used as a blank control group. Compounds $9 \mathbf{9 a}, \mathbf{9 c}, \mathbf{9 j}, \mathbf{1 0 h}$ and $\mathbf{1 0 i}$ displayed more potent insecticidal activity than toosendanin. For example, the final mortality rates (FMRs) of 9a, 9c, 9j, 10h and $\mathbf{1 0 i}$ were $57.1 \%$, $50.0 \%, 50.0 \%, 50.0 \%$, and 57.1\%, respectively; whereas the FMRs of 1 and toosendanin were $42.9 \%$ and $46.4 \%$, respectively. The

Table 1 Growth inhibitory activity of compounds $1,4-6,8 b-j, 9 a-j$, $10 a-j, 11$ and 12 against $M$. separata on leaves treated with a concentration of $1 \mathrm{mg} \mathrm{mL}^{-1}$

\begin{tabular}{|c|c|c|c|}
\hline \multirow[b]{2}{*}{ Compound } & \multicolumn{3}{|c|}{ Corrected mortality rate (mean $\pm \mathrm{SD}, \%$ ) } \\
\hline & 10 days & 20 days & 35 days \\
\hline 1 & $10.0 \pm 0$ & $13.3 \pm 3.3$ & $42.9 \pm 3.3$ \\
\hline 4 & $13.3 \pm 3.3$ & $26.7 \pm 3.3$ & $39.3 \pm 3.3$ \\
\hline 5 & $13.3 \pm 3.3$ & $13.3 \pm 3.3$ & $42.9 \pm 3.3$ \\
\hline 6 & $23.3 \pm 3.3$ & $33.3 \pm 3.3$ & $46.4 \pm 5.8$ \\
\hline $8 \mathbf{b}$ & $6.7 \pm 3.3$ & $10.0 \pm 0$ & $35.7 \pm 5.8$ \\
\hline $8 c$ & $20.0 \pm 0$ & $26.7 \pm 3.3$ & $42.9 \pm 3.3$ \\
\hline 8d & $16.7 \pm 3.3$ & $16.7 \pm 3.3$ & $21.4 \pm 3.3$ \\
\hline $8 \mathbf{e}$ & $10.0 \pm 0$ & $13.3 \pm 3.3$ & $21.4 \pm 3.3$ \\
\hline $8 f$ & $10.0 \pm 5.8$ & $6.7 \pm 6.7$ & $35.7 \pm 0$ \\
\hline $8 \mathrm{~g}$ & $26.7 \pm 3.3$ & $33.3 \pm 6.7$ & $39.3 \pm 3.3$ \\
\hline $8 \mathbf{h}$ & $16.7 \pm 3.3$ & $16.7 \pm 3.3$ & $42.9 \pm 3.3$ \\
\hline $8 \mathbf{i}$ & $26.7 \pm 3.3$ & $33.3 \pm 3.3$ & $42.9 \pm 3.3$ \\
\hline $8 \mathbf{j}$ & $13.3 \pm 3.3$ & $13.3 \pm 3.3$ & $32.1 \pm 3.3$ \\
\hline 9a & $20.0 \pm 5.8$ & $23.3 \pm 6.7$ & $57.1 \pm 0$ \\
\hline $9 b$ & $36.7 \pm 3.3$ & $36.7 \pm 3.3$ & $46.4 \pm 5.8$ \\
\hline $9 c$ & $16.7 \pm 3.3$ & $20.0 \pm 5.8$ & $50.0 \pm 3.3$ \\
\hline 9d & $13.3 \pm 3.3$ & $13.3 \pm 3.3$ & $46.4 \pm 5.8$ \\
\hline $9 e$ & $30.0 \pm 0$ & $33.3 \pm 3.3$ & $42.9 \pm 3.3$ \\
\hline 9f & $6.7 \pm 3.3$ & $13.3 \pm 3.3$ & $32.1 \pm 6.7$ \\
\hline $9 \mathrm{~g}$ & $26.7 \pm 3.3$ & $26.7 \pm 3.3$ & $39.3 \pm 3.3$ \\
\hline $9 h$ & $13.3 \pm 3.3$ & $16.7 \pm 3.3$ & $35.7 \pm 0$ \\
\hline $9 \mathrm{i}$ & $3.3 \pm 3.3$ & $10.0 \pm 0$ & $46.4 \pm 5.8$ \\
\hline 9j & $10.0 \pm 0$ & $10.0 \pm 0$ & $50.0 \pm 3.3$ \\
\hline $10 a$ & $13.3 \pm 3.3$ & $13.3 \pm 3.3$ & $46.4 \pm 5.8$ \\
\hline $10 \mathrm{~b}$ & $10.0 \pm 0$ & $13.3 \pm 3.3$ & $35.7 \pm 5.8$ \\
\hline $10 \mathrm{c}$ & $20.0 \pm 0$ & $23.3 \pm 3.3$ & $42.9 \pm 3.3$ \\
\hline 10d & $6.7 \pm 3.3$ & $6.7 \pm 3.3$ & $39.3 \pm 3.3$ \\
\hline $10 \mathrm{e}$ & $20.0 \pm 0$ & $30.0 \pm 0$ & $42.9 \pm 3.3$ \\
\hline $10 f$ & $26.7 \pm 3.3$ & $26.7 \pm 3.3$ & $42.9 \pm 3.3$ \\
\hline $10 \mathrm{~g}$ & $23.3 \pm 3.3$ & $30.0 \pm 0$ & $42.9 \pm 3.3$ \\
\hline $10 \mathrm{~h}$ & $13.3 \pm 3.3$ & $26.7 \pm 3.3$ & $50.0 \pm 3.3$ \\
\hline $10 \mathrm{i}$ & $13.3 \pm 3.3$ & $20.0 \pm 0$ & $57.1 \pm 5.8$ \\
\hline $10 \mathrm{j}$ & $26.7 \pm 3.3$ & $30.0 \pm 0$ & $46.4 \pm 0$ \\
\hline 11 & $6.7 \pm 3.3$ & $13.3 \pm 3.3$ & $25.0 \pm 5.8$ \\
\hline 12 & $16.7 \pm 6.7$ & $23.3 \pm 3.3$ & $42.9 \pm 3.3$ \\
\hline Toosendanin & $6.7 \pm 3.3$ & $16.7 \pm 3.3$ & $46.4 \pm 5.8$ \\
\hline Blank control & $0 \pm 0$ & $0 \pm 0$ & $6.7 \pm 3.3$ \\
\hline
\end{tabular}


symptoms for the treated $M$. separata during the larval, pupation and adult periods were observed by the same way as our previous reports. ${ }^{14,15,17}$ For example, the dead larvae with thin and wrinkled bodies, the malformed and dead pupae, and the malformed moths also appeared during the above three stages.

Additionally, the structure-activity relationships of tested compounds were also observed. When compared with toosendanin, all esters (8b-j) and oxime sulfonates (11 and 12) exhibited less potent insecticidal activity. To $5^{\prime}$-oxime esters $(\mathbf{9 a}-\mathbf{j})$, introduction of the electron-withdrawing groups on the phenyl of 9b usually resulted in the less active compounds (except 9i,j); for instance, the FMRs of 9e-h were $42.9 \%, 32.1 \%$, $39.3 \%$ and $35.7 \%$, respectively. On the contrary, to $2^{\prime}$-oxime esters (10a-j), compounds containing the electron-withdrawing groups generally showed more potent insecticidal activity than those containing the electron-donating ones. For example, the FMRs of 10e-j were all greater than $42.9 \%$, especially the FMR of $10 \mathrm{i}$ was $57.1 \%$. Previously, we noticed that introduction of a (1naphthylacetyl)oxy group at the $\mathrm{C}-4$ position of the podophyllotoxin derivatives could lead to promising compounds $;^{18-20}$ in this paper, introduction of a (1-naphthylacetyl)oxy group to 5 or 6 also produced the potent compounds 9a and 10a, respectively. So this suggested that the (1-naphthylacetyl)oxy group could be introduced into other natural products in the future.

\section{Conclusions}

In summary, we have semisynthesized a series of esters and oxime esters/sulfonates from furyl-ring-based acetylation derivatives of fraxinellone as pesticidal agents. Especially the steric structures of five products were assigned by X-ray analysis data. Their insecticidal activity was evaluated against a cropthreatening agricultural insect pest, M. separata. Among all derivatives, especially compounds $9 \mathbf{a}$ and $10 \mathbf{i}$ exhibited the most promising pesticidal activity. Their structure-activity relationships were also observed. It will pave the way for further structural modifications of fraxinellones as biorenewable pesticidal agents for agriculture.

\section{Acknowledgements}

The present research was partly supported by National Natural Science Foundation of China (No. 31672071), and Special Funds of Central Colleges Basic Scientific Research Operating Expenses (No. 2452015096) to H. X.

\section{Notes and references}

1 K. Muralidharan and I. C. Pasalu, Crop Prot., 2006, 25, 409417.

2 J. Y. Sun, P. Liang and X. W. Gao, Pest Manage. Sci., 2012, 68, 285-289.

3 J. Zeng, Y. Jiang and J. Liu, Plant Prot., 2013, 39, 117-121.

4 X. L. Wang, S. W. Wu, W. Y. Gao and Y. D. Wu, J. Econ. Entomol., 2016, 109, 68-74.

5 D. G. Heckel, Science, 2012, 337, 334-338.

6 M. Q. Yu, G. Liu, Y. Y. Zhang, F. Tao, M. Xu and H. Xu, Sci. Rep., 2016, 6, 33062.

7 K. M. Mousa, M. M. Elsharkawy, I. A. Khodeir, T. N. ElDakhakhni and A. E. Youssef, Egypt. J. Biol. Pest Control, 2014, 24, 347-351.

8 X. F. Wu, Z. J. Ouyang, L. L. Feng, G. Chen, W. J. Guo, Y. Shen, X. D. Wu, Y. Sun and Q. Xu, Toxicol. Appl. Pharmacol., 2014, 281, 146-156.

9 J. S. Yoon, H. Yang, S. H. Kim, S. H. Sung and Y. C. Kim, J. Mol. Neurosci., 2010, 42, 9-16.

10 Z. L. Liu, S. H. Ho and S. H. Goh, Insect Sci., 2009, 16, 147-155.

11 M. Lv, W. J. Wu and H. X. Liu, Toxins, 2014, 6, 2708-2718.

12 M. Lü, W. J. Wu and H. X. Liu, Pestic. Biochem. Physiol., 2010, 98, 263-268.

13 M. Lv, W. J. Wu and H. X. Liu, Pestic. Biochem. Physiol., 2008, 90, 114-118.

14 Q. Li, X. B. Huang, S. C. Li, J. C. Ma, M. Lv and H. Xu, J. Agric. Food Chem., 2016, 64, 5472-5478.

15 Y. Guo, Y. Yan, X. Yu, Y. Wang and H. Xu, J. Agric. Food Chem., 2012, 60, 7016-7021.

16 Y. Guo, R. Yang and H. Xu, Sci. Rep., 2016, 6, 35321.

17 X. Yu, D. Shi, X. Zhi, Q. Li, X. Yao and H. Xu, RSC Adv., 2015, 5, 31700-31707.

18 H. Xu, X. Xiao, X. F. Zhao, Y. Guo and X. J. Yao, Bioorg. Med. Chem. Lett., 2011, 21, 4008-4012.

19 Z. Che, X. Yu, X. Y. Zhi, L. L. Fan and H. Xu, J. Agric. Food Chem., 2013, 61, 8148-8155.

20 L. L. Fan, Y. Guo, X. Y. Zhi, X. Yu and H. Xu, J. Agric. Food Chem., 2014, 62, 3726-3733. 